\title{
RESOLVENT OPERATORS FOR INTEGRAL EQUATIONS IN A BANACH SPACE
}

BY

\author{
R. C. GRIMMER
}

\begin{abstract}
Conditions are given which ensure the existence of a resolvent operator for an integrodifferential equation in a Banach space. The resolvent operator is similar to an evolution operator for nonautonomous differential equations in a Banach space. As in the finite dimensional case, this operator is used to obtain a variation of parameters formula which can be used to obtain results concerning the asymptotic behaviour of solutions and weak solutions.
\end{abstract}

1. Introduction. In this paper we shall be concerned with the integrodifferential equation

$$
\begin{gathered}
x^{\prime}(t)=A(t) x(t)+\int_{0}^{t} B(t, s) x(s) d s+f(t), \\
x(0)=x_{0} \in X, \quad t \geqslant 0,
\end{gathered}
$$

in a Banach space $X . A(t)$ and $B(t, s)$ are closed linear operators on $X$ with fixed domain which we will denote $D(A)$ while the function $f: R^{+} \rightarrow X$ is continuous. In the convolution case where $A(t) \equiv A$ and $B(t, s) \equiv B(t-s)$ this equation has been studied by numerous authors under various hypotheses concerning $A$ and $B$. Of interest to us here are the papers by Chen and Grimmer [2], Hannsgen [10,11], Miller [14, 15], and Miller and Wheeler [17, 18]. For the nonconvolution case, the papers by Chen and Grimmer [3] and Friedman and Shinbrot [4] are of particular importance to the problem studied here. If $X$ is finite dimensional the work of Grossman and Miller [8] is of significance to us as they develop perturbation theory for (VE) using the resolvent operator for (VE). It is this theory which shall be developed for (VE) when $X$ is not finite dimensional.

The resolvent operator will satisfy an equation like equation (A) of Grossman and Miller pointwise on $D(A)$. It will also resemble an evolution operator for a nonautonomous linear differential equation in a Banach space (cf. e.g. Tanabe [21]). It will not, however, be an evolution operator because it will not satisfy an evolution or semigroup property. Because a number of results follow directly from the definition of the resolvent operator we shall first hypothesize the existence of a resolvent and obtain the variation of parameters formula along with proving

Received by the editors March 2, 1981 and, in revised form, August 20, 1981.

1980 Mathematics Subject Classification. Primary 45D05, 45K05, 45J05, 45N05; Secondary 34A10, 34G10, 47D05.

'This research was done while the author was on leave and a guest at the Control Theory Centre, University of Warwick, Coventry, England. 
uniqueness of such an operator. In $\S 3$, the existence of a resolvent operator will be proved under fairly general circumstances. In particular, the conditions which yielded well-posedness for (VE) in [3] will be sufficient to prove the existence of a resolvent operator. This resolvent operator will be the resolvent operator obtained by Miller [14] in the convolution case where it was assumed $B$ mapped the domain of $A^{2}$ into the domain on $A$. It is also the resolvent operator obtained by Friedman and Shinbrot [4] in the case when $A(t)$ is parabolic and $B(t, s)=-h^{\prime}(t-s) A(s)$. The results obtained here require a bit more smoothness than required by Friedman and Shinbrot. Here $h^{\prime}(t)$ and $A(t) x, x \in D(A)$, are required to be strongly continuously differentiable, while in [4], $h^{\prime}$ is absolutely continuous and $A(t)$ satisfies a Hölder condition on $D(A)$. However, the results obtained here allow more general forms of $B(t, s)$ and include the hyperbolic case, cf. $[3, \S 6]$.

In $\$ 4$ asymptotic results and perturbations are considered. A result is obtained for the convolution case which guarantees that the resolvent operator $R(t)$ satisfies $\|R(t)\| \leqslant M e^{-\alpha t}$ for some constants $M \geqslant 1$ and $\alpha>0$. This result is then applied to an equation which arises in a number of areas and in which $x^{\prime}=A x$ is a damped wave equation.

Finally, in $\$ 5$ we show that we may use our earlier work to obtain a resolvent operator for the integral equation

$$
x(t)=\int_{0}^{t} a(t, s) x(s) d s+f(t) .
$$

It is shown that under certain conditions the variation of parameters equation is valid here also. The results obtained here are not as satisfactory as the results obtained for (VE), however, because in general the resolvent for (IE) is not a bounded operator. For related work cf. Friedman and Shinbrot [4] and also Grimmer and Miller [5, 6].

2. Resolvent operators. We shall assume for each $t \in[0, T], T>0$, that $A(t)$ is a closed linear operator with dense domain $D(A)$ which is independent of $t, 0 \leqslant t \leqslant T$. Also, for $0 \leqslant s \leqslant t \leqslant T, B(t, s)$ is a closed linear operator with domain at least $D(A)$. Suppose $Y$ is the Banach space formed from $D(A)$ with the graph norm $\|y\|_{Y}=\|A(0) y\|+\|y\|$ where \|\| is the norm on $X$. As $A(t)$ and $B(t, s)$ are closed operators it follows that $A(t)$ and $B(t, s)$ are in the set of bounded operators from $Y$ to $X, \mathscr{B}(Y, X)$, for $0 \leqslant t \leqslant T$ and $0 \leqslant s \leqslant t \leqslant T$, respectively. Assume further that $A(t)$ and $B(t, s)$ are continuous on $0 \leqslant t \leqslant T$ and $0 \leqslant s \leqslant t \leqslant T$, respectively, into $\mathscr{B}(Y, X)$.

As we shall be interested primarily in strong solutions, we shall restrict the initial condition for (VE) to $x_{0} \in D(A)$.

Definition 2.1. By a solution $x(t)$ of (VE) on $[0, T]$ with $x(0)=x_{0} \in D(A)$ we mean a function $x \in C([0, T], Y) \cap C^{1}([0, T], X)$ such that (VE) is satisfied for $t \in[0, T]$.

We remark that this is a slight change from the definition given in [3]. It follows, however, that $A(t) x(t) \in C([0, T], X)$ which was what was required earlier. The solutions obtained in [3] do satisfy the above definition. 
DEFINITION 2.2. A resolvent operator for (VE) is a bounded operator valued function $R(t, s) \in \mathscr{B}(X)$ with $0 \leqslant s \leqslant t \leqslant T$, having the following properties:

(a) $R(t, s)$ is strongly continuous in $s$ and $t, R(s, s)=I, 0 \leqslant s \leqslant T,\|R(t, s)\| \leqslant$ $M e^{\beta(t-s)}$ for some constants $M$ and $\beta$.

(b) $R(t, s) Y \subset Y, R(t, s)$ is strongly continuous in $s$ and $t$ on $Y$.

(c) For each $x \in D(A), R(t, s) x$ is strongly continuously differentiable in $t$ and $s$ and

$$
\begin{aligned}
& \frac{\partial R}{\partial t}(t, s) x=A(t) R(t, s) x+\int_{s}^{t} B(t, r) R(r, s) x d r \\
& \frac{\partial R}{\partial s}(t, s) x=-R(t, s) A(s) x-\int_{s}^{t} R(t, r) B(r, s) x d r
\end{aligned}
$$

with $(\partial R / \partial t)(t, s) x$ and $(\partial R / \partial s)(t, s) x$ strongly continuous on $0 \leqslant s \leqslant t \leqslant T$.

We remark that if $A(t)$ and $B(t, s)$ are defined for $0 \leqslant t<\infty$ respectively then the above definition is easily extended by writing $0 \leqslant t<\infty$ and $0 \leqslant s \leqslant t<\infty$ at the appropriate places instead of $0 \leqslant t \leqslant T$ and $0 \leqslant s \leqslant t \leqslant T$, respectively.

We first note that there can be at most one resolvent operator.

THEOREM 2.3. There exists at most one resolvent operator.

Proof. Suppose $R(t, s)$ and $R_{1}(t, s)$ are resolvent operators. Let $x \in D(A)$. Then from (c)

$$
\begin{aligned}
R_{1}(t, s) x-R(t, s) x= & \int_{s}^{t} \frac{\partial}{\partial r}\left[R(t, r) R_{1}(r, s) x\right] d r \\
= & -\int_{s}^{t} \int_{r}^{t} R(t, u) B(u, r) R_{1}(r, s) x d u d r \\
& +\int_{s}^{t} \int_{s}^{r} R(t, r) B(r, u) R_{1}(u, s) x d u d r .
\end{aligned}
$$

As $x \in D(A), R(t, r) B(r, u) R_{1}(u, s) x$ is continuous in $r$ and $u$. It follows then from Fubini's theorem that $R_{1}(t, s) x-R(t, s) x=0,0 \leqslant s \leqslant t \leqslant T$, for all $x \in D(A)$. As $D(A)$ is dense in $X$ and $R_{1}(t, s)-R(t, s)$ is a bounded operator, the result follows.

Theorem 2.4. If $A(t) \equiv A$ and $B(t, s) \equiv B(t-s)$ and there exists a resolvent operator $R(t, s)$, then $R(t, s)=R(t-s)$.

Proof. Let $\tau$ be fixed with $0<\tau<T$. Consider $Q(t, s)=R(t+\tau, s+\tau)$ on $0 \leqslant s \leqslant t \leqslant T-\tau$. Clearly $Q(t, s)$ satisfies (a) and (b) of Definition 2.1. Also, if $x \in D(A), Q(t, s) x$ is strongly continuously differentiable in $t$ and $s, 0 \leqslant s \leqslant t \leqslant T$ $-\tau$. Further

$$
\begin{aligned}
\frac{\partial Q}{\partial t}(t, s) x & =A R(t+\tau, s+\tau) x+\int_{s+\tau}^{t+\tau} B(t+\tau-r) R(r, s+\tau) x d \tau \\
& =A Q(t, s) x+\int_{s}^{t} B(t-u) Q(u, s) x d u .
\end{aligned}
$$

Similarly, the second equation in (c) is also valid for $Q$. Thus, on $0 \leqslant s \leqslant t \leqslant T-\tau$ we must have $Q(t, s)=R(t, s)$ by uniqueness of the resolvent on $[0, T-\tau]$. Now if 
$t=s, R(t, s)=R(t-s, 0)$ is clear and if $t>s>0$ we have $R(t, s)=R(t-s, 0)$ by taking $s=\tau$.

THEOREM 2.5. Suppose a resolvent operator exists for (VE). If $x(t)$ is a solution of (VE) then

$$
x(t)=R(t, 0) x_{0}+\int_{0}^{t} R(t, s) f(s) d s .
$$

Proof. Consider

$$
\begin{aligned}
x(t)-R(t, 0) x_{0}- & \int_{0}^{t} R(t, s) f(s) d s \\
& =\int_{0}^{t} \frac{\partial}{\partial s}[R(t, s) x(s)] d s-\int_{0}^{t} R(t, s) f(s) d s .
\end{aligned}
$$

Using the fact that $x(t)$ is a solution of (VE) and part (c) it follows that the above can be written

$$
\int_{0}^{t} \int_{0}^{s} R(t, s) B(s, v) x(v) d v d s-\int_{0}^{t} \int_{s}^{t} R(t, u) B(u, s) x(s) d u d s .
$$

It follows from the definition of a solution of (VE) that $R(t, u) B(u, s) x(s)$ is continuous. Hence, by Fubini's theorem the result follows.

This result is the standard Variation of Parameters formula for (VE). Because $X$ is infinite dimensional (VP) need not actually be a solution, just as in the case of differential equations. In general, even if $x_{0} \in D(A)$, (VP) will not necessarily yield a solution. Accordingly, we make the following definition.

Definition 2.6. For $x_{0} \in X$ and continuous $f:[0, T] \rightarrow X$ we say that

$$
x(t)=R(t, 0) x_{0}+\int_{0}^{t} R(t, s) f(s) d s
$$

is a weak solution of (VE) on $[0, T]$.

An obvious problem is to determine when weak solutions are strong solutions. A partial answer is given by the following theorem. We shall consider this problem again in the next section.

THEOREM 2.7. Suppose a resolvent operator exists for (VE). If $f \in C([0, T], Y)$ then

$$
v(t)=\int_{0}^{t} R(t, s) f(s) d s
$$

is a solution of (VE).

Proof. From Definition 2.2 we see that

$$
\begin{aligned}
v^{\prime}(t) & =R(t, t) f(t)+\int_{0}^{t} \frac{\partial R}{\partial t}(t, s) f(s) d s \\
& =f(t)+\int_{0}^{t} A(t) R(t, s) f(s) d s+\int_{0}^{t} \int_{s}^{t} B(t, u) R(u, s) f(s) d u d s .
\end{aligned}
$$

As $A(t)$ and $B(t, s)$ are closed operators,

$$
v^{\prime}(t)=f(t)+A(t) v(t)+\int_{0}^{t} B(t, u) v(u) d u
$$


3. Existence of resolvent operators. In this section it is shown that under fairly general conditions (VE) has a resolvent operator as defined by Definition 2.2. It should be noted that it will not be assumed that $A(t)$ generates an analytic semigroup.

To obtain the existence of a resolvent operator, (VE) shall be reformulated as a differential equation. This differential equation will then be shown to have an evolution operator $U(t, s)$. From $U(t, s)$ we shall extract the resolvent operator.

To facilitate our analysis, it shall be assumed that the function space $\mathscr{F}$ is a subspace of the set of bounded uniformly continuous fuctions on $R^{+}$into $X, B U$. It is assumed that $\mathscr{F}$ is a Banach space with a norm stronger than the sup norm on $B U$. Further, it is required that, for each $t \geqslant 0, B(t+\cdot, t) x \in \mathcal{F}$ for every $x \in D(A)$ where $(B(t+\cdot, t) x)(s)=B(t+s, t) x$ for $s \geqslant 0$. This then defines an operator $B(t)$ from $X$ to $\mathscr{F}$ which has domain $D(A)$. It is further assumed that $\{T(t)\}_{t \geqslant 0}$ defined by $T(t) f(s)=f(t+s)$ is a $C_{0}$ semigroup on $\mathcal{F}$ with generator $D_{s}$ on domain $D\left(D_{s}\right)$.

Let $Z=X \times \mathscr{F}$ with the norm $\|(x, y)\|=\|x\|+\|y\|_{\mathscr{F}}$. We will write elements of $Z$ as $(x, y)^{*}$ where $^{*}$ indicates transpose. On the Banach space $Z$ the differential equation

$$
z^{\prime}(t)=C(t) z(t), \quad z(0)=z_{0} \in D(C),
$$

is of importance in the study of (VE). Here $D(C)=D(A) \times D\left(D_{s}\right)$ is the domain of $C(t)$ for $0 \leqslant t<\infty$ and $C(t)$ is given by

$$
C(t)=\left[\begin{array}{ll}
A(t) & \delta_{0} \\
B(t) & D_{s}
\end{array}\right]
$$

where $\delta_{0}: \mathscr{F} \rightarrow X$ is defined by $\delta_{0} f=f(0)$. To obtain an evolution operator for (DE) it will be necessary to assume that for each fixed $t, t \geqslant 0, A(t)$ generates a $C_{0}$ semigroup. The concept of a stable family of generators will also be needed.

Definition 3.1 [21, p. 93]. If $\{A(t)\}, 0 \leqslant t \leqslant T$, is a family of generators of $C_{0}$ semigroups, $\{A(t)\}$ is called stable if there are real constants $M \geqslant 1$ and $\beta$ so that

$$
\left\|\prod_{j=1}^{k}\left(A\left(t_{j}\right)-\lambda I\right)^{-1}\right\| \leqslant M(\lambda-\beta)^{-k}
$$

for all $\lambda>\beta, 0 \leqslant t_{1} \leqslant t_{2} \leqslant \cdots \leqslant t_{k} \leqslant T, k=1,2 \ldots$, where the product is taken to mean

$$
\left(A\left(t_{k}\right)-\lambda I\right)^{-1}\left(A\left(t_{k-1}\right)-\lambda I\right)^{-1} \cdots\left(A\left(t_{1}\right)-\lambda\right)^{-1} .
$$

If $A(t)$ is constant or if $A(t)$ generates a semigroup $\left\{S_{t}(s)\right\}$ with $\left\|S_{t}(s)\right\| \leqslant e^{\beta s}$ for each $t \geqslant 0$ then $\{A(t)\}$ is stable. Other examples also exist.

To obtain the existence of a resolvent operator we shall need a number of hypotheses.

(H0). $\{A(t)\}, 0 \leqslant t \leqslant T$, is a stable family of generators such that $A(t) x$ is strongly continuously differentiable on $[0, T]$ for $x \in D(A)$. In addition, $B(t) x$ is strongly continuously differentiable on $[0, T]$ for $x \in D(A)$.

Note that (H0) guarantees that $A(t)$ and $B(t)$ are continuous on $[0, T]$ into $\mathscr{B}(Y, X)$ and $\mathscr{B}(Y, \mathscr{F})$ respectively. In fact, $A(t)$ is Lipschitzian in $t$ as $A^{\prime}(t)$ is in 
乌 $(Y, X)$ and an application of the Uniform Boundedness Principle yields that $A^{\prime}(t)$ is bounded uniformly in $t$ as an element of $\mathscr{B}(Y, X)$. Similar remarks are valid for $B(t)$.

(H1). $B(t)$ is continuous on $[0, \infty)$ into $\Re(Y, \mathscr{F})$.

(H2). $B(t): Y \rightarrow D\left(D_{s}\right)$ for all $t \geqslant 0$.

(H3). $D_{s} B(t)$ is continuous on $[0, \infty)$ into $\mathscr{B}(Y, \mathscr{F})$.

It should be noted that in the convolution case when $A(t) \equiv A$ and $B(t, s) \equiv$ $B(t-s)$, the operator $B(t) \in \mathscr{B}(Y, \mathcal{F})$ is constant. Thus, $(\mathrm{H} 0)$ and $(\mathrm{H} 1)$ are automatically satisfied if $A$ generates a semigroup. If (H2) is valid, (H3) will follow automatically as $D_{s}$ is closed. Also we note that $(\mathrm{H} 1)-(\mathrm{H} 3)$ are somewhat simplified versions of the conditions $(\mathrm{H} 1)-(\mathrm{H} 3)$ of [3]. The conditions are simpler here because $\mathscr{F}$ consists of continuous functions only. We also remark that in [3] the operator $C(t)$ had the form

$$
\left[\begin{array}{ccc}
0 & A(t) & 0 \\
0 & A(t) & \delta_{0} \\
0 & B(t) & D_{s}
\end{array}\right]
$$

This has been reduced to the form that is given here because the problem has been slightly reformulated. The arguments in [3] are valid in this case also. Further remarks on this will be made when necessary.

Definition 3.2. Let $Z_{1}=D(A) \times D\left(D_{s}\right)$ be normed with $\|(x, y)\|_{1}=\|x\|_{Y}+$ $\|y\|_{D}$ where $\|\cdot\|_{D}$ is the graph norm of $D_{s}$. By a solution of (DE) we mean a function $Z \in C\left([0, T], Z_{1}\right) \cap C^{1}([0, T], Z)$ such that $z(0)=z_{0}$, and (DE) is satisfied on $[0, T]$.

As $B(0)$ and $\delta_{0}$ are bounded operators $Y \rightarrow X$ and $\mathscr{F}$, respectively, repeated application of the triangle inequality shows that $\|\cdot\|_{1}$ is equivalent to the graph norm of $C(0)$ on $D(C)$. Also, $C(t)$ is continuous on $[0, T]$ into $\Re\left(Z_{1}, Z\right)$ and $C(t) z$ is strongly continuously differentiable for $z \in D(C)$ if $(\mathrm{H} 0)$ is valid.

In order to get a resolvent operator it will be necessary to establish the relationship between the systems

$$
\begin{gathered}
x^{\prime}(t)=A(t) x(t)+\int_{r}^{t} B(t, u) x(u) d u+f(t), \\
x(r)=x_{0}, \quad 0 \leqslant r \leqslant t \leqslant T,
\end{gathered}
$$

and

$(\mathrm{DE})_{r}$

$$
z^{\prime}(t)=C(t) z(t), \quad z(r)=z_{0}, \quad 0 \leqslant r \leqslant t \leqslant T .
$$

THEOREM 3.3. Suppose $(\mathrm{H} 1)-(\mathrm{H} 3)$ are valid and $f \in D\left(D_{s}\right)$. If $x(t)$ is a solution of $(\mathrm{VE})_{r}$ then $z(t)=(x(t), y(t))^{*}$ is a solution of $(\mathrm{DE})_{r}$ with $z_{0}=\left(x_{0}, f_{r}\right)^{*}$ where $y(t)=T(t-r) f_{r}+\int_{r}^{t} T(t-u) B(u) x(u) d u$. Here $T(t)$ is the translation semigroup generated by $D_{s}$ and $f_{r}=T(r) f$.

Conversely, if $(\mathrm{H1})$ is valid and $z(t)$ is a solution of $(\mathrm{DE})_{r}$ with $z(r)=\left(x_{0}, f_{r}\right)^{*}$, $z(t)=(x(t), y(t))^{*}, r \leqslant t \leqslant T$, then $x(t)$ is a solution of $(\mathrm{VE})_{r}$. 
Proof. Suppose $x(t)$ is a solution of (VE) $r$. Then $B(t) x(t)$ is continuous on $[r, T]$ into $\mathscr{F}$ by $(\mathrm{H} 1)$. Also, as $(\mathrm{H} 2)$ and $(\mathrm{H} 3)$ are valid, $D_{s} B(t) x(t)$ is continuous and so the equation $y^{\prime}(t)=D_{s} y(t)+B(t) x(t), y(r)=f_{r}$, has the solution

$$
y(t)=T(t-r) f_{r}+\int_{r}^{t} T(t-u) B(u) x(u) d u .
$$

At $\tau \geqslant 0$,

$$
\begin{aligned}
y(t)(\tau) & =T(t-r) f_{r}(\tau)+\int_{r}^{t} T(t-u) B(u+\tau, u) x(u) d u \\
& =f(t+\tau)+\int_{r}^{t} B(t+\tau, u) x(u) d u
\end{aligned}
$$

Hence, $\delta_{0} y(t)=f(t)+\int_{r}^{t} B(t, u) x(u) d u$ and $z(t)=(x(t), y(t))^{*}$ is a solution of $(\mathrm{DE})_{r}$ on $[r, T]$ with the prescribed initial conditions.

Now if $z(t)$ is a solution of (DE) $)_{r}$ on $[r, T]$ with $z(r)=\left(x_{0}, f_{r}\right)^{*}$, for $r \leqslant t \leqslant T$ we have, with $z(t)=(x(t), y(t))^{*}$,

$$
x^{\prime}(t)=A(t) x(t)+\delta_{0} y(t), \quad y^{\prime}(t)=D_{s} y(t)+B(t) x(t) .
$$

As $z \in C\left([r, T], Z_{1}\right) \cap C^{1}([r, T], Z)$ it follows that $x \in C([r, T], Y) \cap$ $C^{1}([r, T], X)$. From $(\mathrm{H} 1), B(t) x(t)$ is a continuous function on $[r, T]$ into $\mathscr{F}$ and so $y(t)$ can be written

$$
y(t)=T(t-r) f_{r}+\int_{r}^{t} T(t-u) B(u) x(u) d u
$$

for $r \leqslant t \leqslant T$. As before, $\delta_{0} y(t)=f(t)+\int_{r}^{t} B(t, u) x(u) d u$ so that $x(t)$ is a solution of $(\mathrm{VE})_{r}$.

It will also be important to relate $(\mathrm{VE})_{r}$ to the equation

$$
z^{\prime}(t)=C(t) z(t)+G(t), \quad z(r)=z_{0}, \quad r \leqslant t \leqslant T,
$$

where $G(t)=(f(t), 0)^{*}$.

TheOREM 3.4. Suppose (H1)-(H3) are valid and $f$ is continuous on $[r, T]$ into $X$. If $x(t)$ is a solution of $(\mathrm{VE})_{r}$ then $z(t)=(x(t), y(t))^{*}$ is a solution of $(\mathrm{G})_{r}$ with $z(r)=\left(x_{0}, 0\right)^{*}$ where $y(t)=\int_{r}^{t} T(t-u) B(u) x(u) d u$. Conversely, suppose (H1) is valid and $f(t)$ is continuous on $[r, T]$ into $X$. If $z(t)$ is a solution of $(G)_{r}$ with $z(r)=\left(x_{0}, 0\right)^{*}$ and $z(t)=(x(t), y(t))^{*}, r \leqslant t \leqslant T$, then $x(t)$ is a solution of (VE) $r$.

As the proof of this result is identical to that of the preceding result, it is omitted.

It is now necessary to state exactly what is meant by the term evolution operator in this paper. Other definitions are possible, cf. e.g. [21] and the references therein.

DEFINITION 3.5. An evolution operator for (DE) is a bounded operator valued function $U(t, s) \in \mathscr{B}(Z)$ with $0 \leqslant s \leqslant t \leqslant T$ which satisfies the following properties:

(a) $U(t, s)$ is strongly continuous in $s$ and $t, U(s, s)=I$, and $\|U(t, s)\| \leqslant M e^{\beta(t-s)}$ for some constants $M$ and $\beta$.

(b) $U(t, s)=U(t, r) U(r, s), 0 \leqslant s \leqslant r \leqslant t \leqslant T$.

(c) $U(t, s) Z_{1} \subset Z_{1}, U(t, s)$ is strongly continuous in $s$ and $t$ on $Z_{1}$. 
(d) For each $z \in Z_{1}, U(t, s) z$ is strongly continuously differentiable in $t$ and $s$ with

$$
\begin{gathered}
(\partial / \partial t) U(t, s) z=C(t) U(t, s) z, \\
(\partial / \partial s) U(t, s) z=-U(t, s) C(s) z .
\end{gathered}
$$

The next result follows from Theorem 3.3 of [3] where a broader class of function spaces $\mathscr{F}$ was considered. As mentioned earlier, the operator $C(t)$ in [3] was

$$
C(t)=\left[\begin{array}{lll}
0 & A(t) & 0 \\
0 & A(t) & \delta_{0} \\
0 & B(t) & D_{s}
\end{array}\right]
$$

defined on $X \times D(A) \times D\left(D_{s}\right) \subset X \times X \times \mathscr{F}$. The only reason for the larger space and different operator was so that a version of Theorem 3.3 could be proven for a larger class of function spaces: Restricting ourselves to spaces $\mathscr{F} \subset B U([0, \infty), X)$, as we have, the arguments in [3] are easily seen to be valid in our setting and we shall feel free to use the results from [3].

THEOREM 3.6. Suppose (H0)-(H3) are valid. Then (DE) has an evolution operator.

Proof. We need only show that there is a constant $\gamma>0$ so that for $0 \leqslant t \leqslant T$,

$$
\|B(t) x\| \leqslant \gamma(\|x\|+\|A(t) x\|)
$$

for all $x \in D(A)$ and the result is immediate from Theorem 3.3 of [3].

From (H1) it follows that there exists a constant $\gamma_{1}>0$ so that

$$
\|B(t) x\| \leqslant \gamma_{1}(\|x\|+\|A(0) x\|) \text {. }
$$

Now if $A(0)(A(t)-\gamma I)^{-1}$ is uniformly bounded on $[0, T]$, for some $\lambda>\beta$,

$$
\|B(t) x\| \leqslant \gamma_{1}\left(\|x\|+\left\|A(0)(A(t)-\lambda I)^{-1}\right\|\|(A(t)-\lambda I) x\|\right)
$$

so that (3.1) is immediate. From the identity

$$
(A(t)-\lambda I)=\left\{I+(A(t)-A(s))(A(s)-\lambda I)^{-1}\right\}(A(s)-\lambda I)
$$

we see that as $A(t)$ is continuous on $[0, T]$ into $\mathscr{B}(Y, X)$, if $(t-s)$ is small, we may use the Neumann series expansion for $\left\{I+(A(t)-A(s))(A(s)-\lambda I)^{-1}\right\}$ to get

$$
(A(t)-\lambda I)^{-1}=(A(s)-\lambda I)^{-1} \sum_{n=0}^{\infty}(-1)^{n}\left\{(A(t)-A(s))(A(s)-\lambda I)^{-1}\right\}^{n} .
$$

We thus obtain the continuity of $(A(t)-\lambda I)^{-1}$ from $[0, T]$ into $\mathscr{B}(X, Y)$. It now follows that $A(0)(A(t)-\lambda I)^{-1}$ is uniformly bounded on $[0, T]$.

The next theorem is the main result of this paper.

THEOREM 3.7. Suppose (H0)-(H3) are valid. Then (VE) has a resolvent operator.

Proof. Let $U(t, s)$ be the evolution operator for (DE) guaranteed by the previous theorem. Writing $U(t, s)$ as $\left[U_{i j}(t, s)\right], i, j=1,2$, it will be shown that $U_{11}(t, s)$ is a resolvent operator for (VE). First note that $U_{11}(t, s) \in \mathfrak{B}(X, X), 0 \leqslant s \leqslant t \leqslant T$, and as $U(s, s)=I_{z}, U_{11}(s, s)=I_{X}$. As $U(t, s)$ is strongly continuous in $t$ and $s, U_{11}(t, s)$ is also. Further, $\left\|U_{11}(t, s)\right\| \leqslant\|U(t, s)\| \leqslant M e^{\beta(t-s)}$. If $x \in Y,(x, 0)^{*} \in Z_{1}$ and 
$U(t, s)(x, 0)^{*} \in Z_{1}$ which implies $U_{11}(t, s) x \in Y$. As $U(t, s)$ is strongly continuous in $s$ and $t$ on $Z_{1}$, the same is true of $U_{11}(t, s)$ on $Y$. We see now that $U_{11}(t, s)$ satisfies (a) and (b) of Definition 2.2. We must now show that $U_{11}$ also satisfies (c).

If $x_{0} \in Y$, then $\left(x_{0}, 0\right)^{*} \in Z_{1}$ and $U(t, s)\left(x_{0}, 0\right)^{*}$ is a solution of (DE) $)_{r}$ and so $U_{11}(t, s) x_{0}$ is a solution of

$$
x^{\prime}(t)=A(t) x(t)+\int_{s}^{t} B(t, r) x(r) d r, \quad x(s)=x_{0},
$$

by Theorem 3.3. That is,

$$
(\partial / \partial t) U_{11}(t, s) x_{0}=A(t) U_{11}(t, s) x_{0}+\int_{s}^{t} B(t, r) U_{11}(r, s) x_{0} d r
$$

for $x_{0} \in Y$.

Now $(\partial / \partial s) U(t, s)\left(x_{0}, 0\right)^{*}=-U(t, s) C(s)\left(x_{0}, 0\right)^{*}$ and so

$$
(\partial / \partial s) U_{11}(t, s) x_{0}=-U_{11}(t, s) A(s) x_{0}-U_{12}(t, s) B(s) x_{0} .
$$

We must determine $U_{12}(t, s)$. If $(0, f)^{*} \in Z_{1}$ then $\left(0, f_{s}\right)^{*} \in Z$ and $U(t, s)\left(0, f_{s}\right)^{*}$ is a solution of $(\mathrm{DE})_{s}$ and so by Theorem 3.3, $U_{12}(t, s) f_{s}$ is a solution of (VE)s. However, by Theorem 3.4, the inhomogeneous equation $(G)_{r}$ has a solution with initial condition $(0,0)^{*}$ which must be $\int_{s}^{t} U(t, u)(f(u), 0)^{*} d u$ as $U$ is an evolution operator for (DE). Thus, $x(t)=\int_{s}^{t} U_{11}(t, u) f(u) d u$. We thus have for $f \in D\left(D_{s}\right)$

$$
U_{12}(t, s) f_{s}=\int_{s}^{t} U_{11}(t, u) f(u) d u
$$

or

$$
U_{12}(t, s) f=\int_{s}^{t} U_{11}(t, u) f(u-s) d u .
$$

As $D\left(D_{s}\right)$ is dense in $\mathscr{F}$ we may extend this formula to all of $\mathscr{F}$.

We now see that

$$
(\partial / \partial s) U_{11}(t, s) x_{0}=-U_{11}(t, s) A(s) x_{0}-\int_{s}^{t} U_{11}(t, r) B(r, s) x_{0} d r
$$

by recalling that $B(s) x_{0}$ as a function of $r$ is given by $\left(B(s) x_{0}\right)(r)=B(r+s, s) x_{0}$. We have now shown that $U_{11}$ satisfies (c) and so $R(t, s) \equiv U_{11}(t, s)$ is a resolvent operator for (VE).

Corollary 3.8. Suppose (H0)-(H3) are valid. If $x_{0} \in D(A)$ and $f \in C^{1}([0, T], X)$ then (VE) has a solution given by

$$
x(t)=R(t, 0) x_{0}+\int_{0}^{t} R(t, s) f(s) d s .
$$

Proof. The existence of the solution has been established in other ways. It follows, however, from Theorem 3.6 and the proof of Theorem 3.2 of [3] that $\{C(t)\}$ is a stable family of generators with common domain such that $C(t) z$ is strongly differentiable for $z \in Z_{1}$. The hypotheses of Theorem 4.5.3 of [21] are thus satisfied with $G(t)=(f(t), 0)^{*}$ and so (DE) has a solution

$$
z(t)=U(t, 0)\left(x_{0}, 0\right)^{*}+\int_{0}^{t} U(t, s)(f(s), 0)^{*} d s
$$


We see then that $x(t)$ must satisfy

$$
x(t)=U_{11}(t, 0) x_{0}+\int_{0}^{t} U_{11}(t, s) f(s) d s .
$$

4. Asymptotic behaviour and perturbations. To determine the asymptotic behaviour of the solutions of (VE) it is extremely useful to have some knowledge of the structure of the resolvent operator. In the finite dimensional case this has been discussed in Grossman and Miller [8] and also in Grimmer and Seifert [7] among others. The main technique is to use known properties of the resolvent along with the variation of parameters formula,

$$
x(t)=R(t, 0) x_{0}+\int_{0}^{t} R(t, s) f(s) d s .
$$

In the infinite dimensional case (VP) yields weak solutions in addition to regular solutions and, hence, the asymptotic behaviour of all weak solutions will be obtained, avoiding the separate question of whether (VP) represents a solution in the usual sense or a weak solution. Clearly, if the asymptotic behaviour of all weak solutions is obtained the same behaviour is valid for all solutions.

Let us consider the convolution integral equation

$$
x^{\prime}(t)=A x(t)+\int_{0}^{t} B(t-s) x(s) d s+f(t), \quad x(0)=x_{0} .
$$

If $A$ generates a semigroup $T_{1}(t)$ with $\left\|T_{1}(t)\right\| \leqslant M e^{\omega t}$ we shall say $A \in G(M, \omega)$. In particular, if $A \in G(M,-\alpha), \alpha>0$, then $A^{-1}$ exists as a bounded operator and $B(t)=B(t) A^{-1} A$. We thus may write $B(t)=F(t) A$ where $F(t)$ is a bounded operator. Also, it will be convenient to consider function spaces other than the space of bounded uniformly continuous functions defined on $[0, \infty)$ into $X, B U([0, \infty), X)$. More specifically, define the space $\mathscr{F}_{\alpha}^{a}, a>0$, by

$$
\mathscr{F}_{\alpha}^{a}=\left\{f \in B U: e^{\alpha t} f(t) \in B U\right\}
$$

with norm $\|f\|=\sup \left\{\left\|a e^{\alpha t} f(t)\right\|: t \geqslant 0\right\}$. Note that on $\mathscr{F}_{\alpha}^{a}$, if $T(t)$ is the translation semigroup generated by $D_{s}$,

$$
\|T(t) f\|=\sup \left\{\left\|a e^{\alpha s} f(t+s)\right\|: s \geqslant 0\right\} \leqslant e^{-\alpha t}\|f\| .
$$

Theorem 4.1. Suppose $A \in G(M,-\alpha), \alpha>0, B(t)=F(t) A$ with $F(t) x \in D\left(D_{s}\right)$ $\subset \mathscr{F}_{\beta}^{a}$ for some $\beta>0$ for each $x \in X$. Further, assume $\left\|a e^{\beta t} F(t)\right\| \leqslant 1$, $\left\|a^{2} e^{\beta t} D_{s} F(t)\right\| \leqslant 1$ and $M / a<\min \{\alpha, \beta\} \equiv \gamma$. Then $R(t)$ decays exponentially. Specifically, $\|R(t)\| \leqslant M \exp (-\gamma+M / a) t$.

Proof. On $Z=X \times \mathscr{F}_{\beta}^{a}$, with norm $\|(x, y)\|=\max \{\|x\|,\|y\|\}$, it is clear that

$$
C_{1}=\left[\begin{array}{cc}
A & 0 \\
0 & D_{s}
\end{array}\right] \in G(M,-\gamma) .
$$

Now, let $P=\left[\begin{array}{cc}I & 0 \\ F & I\end{array}\right]$ and define a new norm on $Z$ by $\|z\|=\left\|P^{-1} z\right\|$. Then if $S(t)$ is generated by $C_{1},\left\|P S(t) P^{-1} z\right\|=\left\|S(t) P^{-1} z\right\| \leqslant M e^{-\gamma t} \| Z_{\|}$. As

$$
K=\left[\begin{array}{ll}
0 & \delta \\
D_{s} F & 0
\end{array}\right]
$$


is a bounded operator, $P C_{1} P^{-1}+K \in G(M,-\gamma+M\|K\|)$. However, $\|K\|=$ $\left\|P^{-1} K\right\| \leqslant \max \left\{\|\delta\|,\left\|D_{s} F\right\|,\|F \delta\|\right\}$. As $\delta: \mathscr{F}_{\beta}^{a} \rightarrow X$ has norm $1 / a$, we see that $\|K\| \leqslant 1 / a$. If $S_{1}(t)$ is the semigroup generated by $P C_{1} P^{-1}+K$, we have $\|R(t)\| \leqslant$ \|I $S_{1}(t) \|$. In particular, $\left\|S_{1}(t)\left(x_{0}, 0\right)^{*}\right\| \|=\max \left\{\left\|R(t) x_{0}\right\|,\left\|S_{21}(t) x_{0}-F R(t) x_{0}\right\|\right\}$ where $S_{21}(t)$ is $\Pi_{2} S(t) \Pi_{1}$ where $\Pi_{1}$ is the projection from $Z$ to $X$ and $\Pi_{2}$ is the projection of $Z$ to $\mathscr{F}_{\beta}^{a}$. The result now follows immediately.

To determine the asymptotic behaviour of the weak solutions of (VE) we note that if the hypotheses of the previous theorem are satisfied then $R(t) x_{0} \rightarrow 0$ as $t \rightarrow \infty$ for each $x_{0} \in X$. Also, if $\rho$ is the operator defined by

$$
\rho(f)(t)=\int_{0}^{t} R(t-u) f(u) d u,
$$

$\rho$ maps each of the following spaces into itself:

$$
\begin{gathered}
B C_{0}=\{f \in B U: f(t) \rightarrow 0 \text { as } t \rightarrow \infty\}, \\
B C_{l}=\left\{f \in B U: \lim _{t \rightarrow \infty} f(t)=l \text { exists }\right\}, \\
A_{\omega}=\left\{f \in B U: f=p+k, p(t+\omega)=p(t), k \in B C_{0}\right\} .
\end{gathered}
$$

Also, $L^{p}, p \geqslant 1$, and $L^{p} \cap B C_{0}$. See [8 and 16] for details.

As an application, let us consider the equation

$$
\begin{aligned}
C \theta^{\prime \prime}(x, t)+\beta(0) \theta^{\prime}(x, t)= & \alpha(0) \Delta \theta(x, t)-\int_{-\infty}^{t} \beta^{\prime}(t-u) \theta^{\prime}(x, u) d u \\
& +\int_{-\infty}^{t} \alpha^{\prime}(t-u) \Delta \theta(x, u) d u+r^{\prime}(x, t)
\end{aligned}
$$

where ${ }^{\prime}=(d / d t)$. This equation arises in the study of heat conduction in materials with memory, cf. $[9,15,18]$. Let us assume that $\Omega$ is a bounded open connected subset of $\mathbf{R}^{3}$ with $C^{\infty}$ boundary. Also, assume $C$ is a positive constant and that $\alpha$ and $\beta$ are in $C^{2}([0, \infty), \mathbf{R})$ with $\alpha(0)$ and $\beta(0)$ positive. If we suppress the dependence on $x \in \Omega$ and assume that $\theta(x, t)$ is known for $t \leqslant 0$ we may pose the problem as

$$
\begin{aligned}
C \theta^{\prime \prime}(t)+\beta(0) \theta^{\prime}(t)= & \alpha(0) \Delta \theta(t)-\int_{0}^{t} \beta^{\prime}(t-u) \theta^{\prime}(u) d u \\
& +\int_{0}^{t} \alpha^{\prime}(t-u) \Delta \theta(u) d u+f(t) .
\end{aligned}
$$

$\Delta$ is the Laplacian on $\Omega$ with boundary condition $\left.\theta\right|_{\Gamma}=0$ and we have the initial conditions

$$
\theta(x, 0)=\theta_{0}(x) \in H^{2}(\Omega) \cap H_{0}^{1}(\Omega), \quad \theta^{\prime}(x, 0)=\theta_{0}^{\prime}(x) \in H_{0}^{1}(\Omega) .
$$

We rewrite this as

$$
\begin{aligned}
\left(\begin{array}{c}
\theta \\
\Phi
\end{array}\right)^{\prime}= & \left(\begin{array}{cc}
0, & I \\
\alpha_{1}(0) \Delta, & -\beta_{1}(0) I
\end{array}\right)\left(\begin{array}{c}
\theta \\
\Phi
\end{array}\right) \\
& +\int_{0}^{t}\left(\begin{array}{cc}
0, & 0 \\
\alpha_{1}^{\prime}(t-u) \Delta, & -\beta_{1}^{\prime}(t-u) I
\end{array}\right)\left(\begin{array}{c}
\theta(u) \\
\Phi(u)
\end{array}\right) d u+\left(\begin{array}{c}
0 \\
f(t)
\end{array}\right) \\
\left(\begin{array}{c}
\theta(0) \\
\Phi(0)
\end{array}\right) & =\left(\begin{array}{c}
\theta_{0} \\
\theta_{0}^{\prime}
\end{array}\right)
\end{aligned}
$$


where $\alpha_{1}=\alpha / C, \beta_{1}=\beta / C$. Letting $\omega=(\theta, \Phi)^{*}$ this can be written as

$$
\omega^{\prime}(t)=A \omega(t)+\int_{0}^{t} F(t-u) A \omega(u) d u+G(t), \quad \omega(0) \in D(A) \subset H .
$$

Here $H$ is the space $H_{0}^{1}(\Omega) \oplus H^{\circ}(\Omega)$ with inner product

$$
\left\langle\left(\theta_{1}, \Phi_{1}\right),\left(\theta_{2}, \Phi_{2}\right)\right\rangle=\int_{\Omega}\left(\operatorname{grad} \theta_{1} \operatorname{grad} \theta_{2}+\Phi_{1} \Phi_{2}\right) d x,
$$

and $A$ has domain $D(A)=\left(H^{2}(\Omega) \cap H_{0}^{1}(\Omega)\right) \oplus H_{0}^{1}(\Omega)$. It follows from Chen [1] that $A$ generates a semigroup $\{T(t)\}$ on $H$ with $\|T(t)\| \leqslant M e^{-\gamma t}$ where $\gamma>0$. In fact, letting $\beta_{0}=\beta_{1}(0)$ and $\gamma_{0}$ the largest eigenvalue of $\alpha(0) \Delta$ in $H_{0}^{1}(\Omega)$, it follows from Pritchard and Zabczyk [20] that

$$
-\gamma \leqslant-2 \beta_{0}\left|\gamma_{0}\right|\left\{4\left|\gamma_{0}\right|+\beta_{0}\left(\beta_{0}+\left|\beta_{0}^{2}+4\right| \gamma_{0}||^{1 / 2}\right)\right\}^{-1} \text {. }
$$

$F(t)$ is given by $F(t)=\left(F_{i j}(t)\right), F_{11}(t) \equiv F_{12}(t) \equiv 0, F_{22}(t)=\left(\alpha_{1}^{\prime}(t) / \alpha_{1}(0)\right) I$, $F_{21}(t)=-\beta_{1}^{\prime}(t) I+\beta_{1}(0) F_{22}(t)$. Assume that $\alpha_{1}^{\prime}(t) e^{\gamma t}, \alpha_{1}^{\prime \prime}(t) e^{\gamma t}, \beta_{1}^{\prime}(t) e^{\gamma t}$, and $\beta_{1}^{\prime \prime}(t) e^{\gamma t}$ are bounded and uniformly continuous.

Define $F_{1}$ and $F_{2}$ by

$$
F_{1}(t)=\max \left\{\left\|F_{22}(t)\right\|,\left\|F_{21}(t)\right\|\right\}
$$

and

$$
F_{2}(t)=\max \left\{\left\|F_{22}^{\prime}(t)\right\|,\left\|F_{21}^{\prime}(t)\right\|\right\} .
$$

If $F_{1}(t) \leqslant e^{-\gamma t} / a$ and $F_{2}(t) \leqslant e^{-\gamma t} / a^{2}$ with $M / a<\gamma$ it follows from Theorem 4.1 that $\|R(t)\| \leqslant M e^{-\eta t}$ where $\eta=\gamma-M / a$.

The existence of a resolvent operator is also useful when considering perturbations of (VE). As an example, we consider the equation

$$
x^{\prime}(t)=A(t) x(t)+\int_{0}^{t} B(t, s) x(s) d s+F(t)+g(x(t), t),
$$

$$
x(0)=x_{0} \in Y,
$$

where $F$ and $g$ are continuous on $[0, \infty)$ and $X \times[0, \infty)$ into $X$ respectively with $A(t)$ and $B(t, s)$ as before. If (PVE) has a solution it must satisfy

$$
x(t)=R(t, 0) x_{0}+\int_{0}^{t} R(t, s) f(s) d s+\int_{0}^{t} R(t, s) g(x(s), s) d s .
$$

If $g$ satisfies a Lipschitz condition, the operator

$$
K x(t)=R(t, 0) x_{0}+\int_{0}^{t} R(t, s) f(s) d s+\int_{0}^{t} R(t, s) g(x(s), s) d s
$$

can be shown to have a fixed point in $C\left(\left[0, T_{0}\right], X\right)$ if $T_{0}$ is chosen sufficiently small. To actually obtain a solution, however, requires further hypotheses.

THEOREM 4.2. Suppose $f \in C([0, \infty), Y), g: Y \times[0, \infty) \rightarrow Y$ is continuous and that $g$ is Lipschitzian in $x \in Y$. Then (PVE) has a solution on $\left[0, T_{0}\right]$ for some $T_{0}>0$.

PRoof. Consider the operator $K$ given above on $C\left(\left[0, T_{0}\right], Y\right)$. As

$$
x_{0}(t)=R(t, 0) x_{0}+\int_{0}^{t} R(t, s) f(s) d s
$$


is in $C\left(\left[0, T_{0}\right], Y\right)$ by Theorem 2.7 , we need only consider $\int_{0}^{t} R(t, s) g(x(s), s) d s$. From Definition 2.2(b) we see that $K$ maps $C\left(\left[0, T_{0}\right], Y\right)$ into itself.

Also, if $\|\cdot\|_{Y}$ is the norm on $Y$ and $x, y \in C\left(\left[0, T_{0}\right], Y\right)$,

$$
\begin{aligned}
\|K x(t)-K y(t)\|_{Y} & \leqslant \int_{0}^{t}\|R(t, s)\|_{Y}\|g(x(s), s)-g(y(s), s)\|_{Y} d s \\
& \leqslant T_{o} R L\|x-y\|_{\infty}
\end{aligned}
$$

if $0 \leqslant t \leqslant T_{0}, R$ is a bound for $R(t, s)$ in $\mathscr{B}(Y, Y), 0 \leqslant s \leqslant t \leqslant T_{0}$, and $L$ is the Lipschitz constant of $g$. Also, by $\|\cdot\|_{\infty}$ is meant the sup norm on $C\left(\left[0, T_{0}\right], Y\right)$. Clearly, if $T_{0}$ is chosen sufficiently small, $K$ has a fixed point $x(t)$ in $C\left(\left[0, T_{0}\right], Y\right)$. Now as $f(t)+g(x(t), t) \in C\left(\left[0, T_{0}\right], Y\right),(\mathrm{PVE})$ has a solution which must be $x(t)$.

REMARK. The above theorem is false with $g: Y \times[0, \infty) \rightarrow X$, for even if $g(x(t), t)=K(t) x(t)$ where $K(t)$ is a bounded linear operator with $K:[0, \infty) \rightarrow$ $\mathscr{B}(X)$ continuous we may not have a solution. In particular, as (VE) is a differential equation when $B \equiv 0$, consider on $X \times X$ the equation $x^{\prime}(t)=A x(t), y^{\prime}(t)=0$, where $A$ generates the $C_{0}$ semigroup $T(t)$. Let $x_{0} \in X$ be such that $T(t) x_{0} \notin D(A)$ for $t \geqslant 0$. Then $\left(0, x_{0}\right)^{*}$ is in the domain of the operator

$$
\left[\begin{array}{ll}
A, & 0 \\
0, & 0
\end{array}\right]
$$

which generates a $C_{0}$ semigroup. However, the initial value problem

$$
\begin{gathered}
x^{\prime}(t)=A x(t)+T(t) y(t), \\
y^{\prime}(t)=0, \quad x(0)=0, \quad y(0)=x_{0},
\end{gathered}
$$

has no solution. If it did have a solution, then $y(t) \equiv x_{0}$ and $x(t)$ would have to satisfy $x(t)=\int_{0}^{t} T(t) x_{0} d s=t T(t) x_{0}$ and so $T(t) x_{0}$ must be differentiable for $t>0$ or $T(t) x_{0} \in D(A)$ for $t>0$.

We also note that the usual modification of the proof of Theorem 4.2 would allow the use of a local Lipschitz condition for $g$.

5. Integral equations. Consider now the integral equation

$$
x(t)=\int_{0}^{t} a(t, s) x(s) d s+f(t) .
$$

Formally differentiating this equation we get

$$
x^{\prime}(t)=a(t, t) x(t)+\int_{0}^{t} \frac{\partial a}{\partial t}(t, s) x(s) d s+f^{\prime}(t), \quad x(0)=f(0) .
$$

If now $A(t) \equiv a(t, t)$ and $B(t, s)=\partial a(t, s) / \partial t$ satisfy $(\mathrm{H} 0)-(\mathrm{H} 3)$ then there is a resolvent operator $R(t, s)$ associated with (5.1). We wish, however, to obtain a resolvent operator for (IE). In the finite dimensional case the resolvent associated with (IE) would satisfy the equations

$$
r(t, s)=-a(t, s)+\int_{s}^{t} r(t, u) a(u, s) d u
$$

and

$$
r(t, s)=-a(t, s)+\int_{s}^{t} a(t, u) r(u, s) d u
$$


Under fairly mild assumptions, the existence of an operator $r(t, s)$ which satisfies these relations can be shown. Also, for certain functions $f(t)$, the variation of parameters formula

$$
x(t)=f(t)-\int_{0}^{t} r(t, u) f(u) d u
$$

does yield a solution of (IE). For a discussion of this in finite dimensions see Miller [16, Chapter IV].

For our purposes here we shall require that $a(t, s)$ be a closed linear operator defined for $0 \leqslant s \leqslant t<\infty$ with dense domain $Y$ which is independent of $(t, s)$. We also ask that $a(t, s) x$ be strongly continuously differentiable in $t$ for $x \in Y$, $0 \leqslant s \leqslant t<\infty$, and that $(\partial / \partial t) a(t, s)$ exists as a closed operator with domain $Y$ for $0 \leqslant s \leqslant t<\infty$. Assume that $a(t, t)$ generates a $C_{0}$ semigroup for each $t$ and with $\lambda>0$ sufficiently large, define $Y_{1}$ by $Y_{1}=(\lambda I-a(0,0))^{-1} Y$. Endow $Y$ with the graph norm of $a(0,0)$ so that $\|y\|_{Y}=\|y\|+\|a(0,0) y\|$. Consider $Y_{1}$ as a subspace of $Y$ and endow $Y_{1}$ with the graph norm of $a(0,0)$ so that $\|y\|_{Y_{1}}=\|y\|+$ $2\|a(0,0) y\|+\left\|a^{2}(0,0) y\right\|$.

In this setting we are able to obtain a resolvent operator for (IE) which mirrors the finite dimensional case.

THEOREM 5.1. Suppose $A(t) \equiv a(t, t)$ and $B(t, s) \equiv(\partial / \partial t) a(t, s)$ satisfy $(\mathrm{H} 0)-(\mathrm{H} 3)$ and that $a(t, s)$ and $B(t, s)$ are continuous on $0 \leqslant s \leqslant t<\infty$ into $\Re\left(Y_{1}, Y\right)$. Then there exists an operator $r(t, s)$ such that $r(t, s) x_{0}$ is strongly continuous in $t$ and $s$ for $x_{0} \in Y$ and which satisfies

$$
\begin{aligned}
& r(t, s) x_{0}=-a(t, s) x_{0}+\int_{s}^{t} r(t, u) a(u, s) x_{0} d u \\
& r(t, s) x_{0}=-a(t, s) x_{0}+\int_{s}^{t} a(t, u) r(u, s) x_{0} d u
\end{aligned}
$$

for every $x_{0} \in Y_{1}$.

Proof. It follows from Theorem 3.7 that the equation

$$
x^{\prime}(t)=A(t) x(t)+\int_{0}^{t} B(t, s) x(s) d s
$$

has a resolvent operator which satisfies Definition 2.2.

Define $r(t, s)$ by $r(t, s)=(\partial / \partial s) R(t, s)$. For $x_{0} \in Y_{1}$,

$$
r(t, s) x_{0}=-R(t, s) a(s, s) x_{0}-\int_{s}^{t} R(t, u) B(u, s) x_{0} d u .
$$

As $x_{0} \in Y_{1}$, we may integrate by parts to get

$$
r(t, s) x_{0}=-a(t, s) x_{0}+\int_{s}^{t} r(t, u) a(u, s) x_{0} d u .
$$


Also, for $x_{0} \in Y_{1}, R(t, s)$ satisfies

$$
\begin{aligned}
R(t, s) x_{0}-x_{0} & =\int_{s}^{t}(\partial / \partial t) R(u, s) x_{0} d u \\
& =\int_{s}^{t} a(u, u) R(u, s) x_{0} d u+\int_{s}^{t} \int_{s}^{u} B(u, v) R(v, s) x_{0} d v d u \\
& =\int_{s}^{t} a(u, u) R(u, s) x_{0} d u+\int_{s}^{t} \int_{v}^{t} B(u, v) R(v, s) x_{0} d u d v
\end{aligned}
$$

Thus,

$$
R(t, s) x_{0}-x_{0}=\int_{s}^{t} a(t, v) R(v, s) x_{0} d v .
$$

Now, notice that it follows from (5.4) that $r(t, s)$ maps $Y_{1}$ into $Y$ and that $r(t, s) x_{0}$ is strongly continuous in $Y, 0 \leqslant s \leqslant t<\infty, x_{0} \in Y_{1}$. Hence, as $x_{0} \in Y_{1}$, it follows from (5.5) that

$$
r(t, s) x_{0}=-a(t, s) x_{0}+\int_{s}^{t} a(t, v) r(v, s) x_{0} d v .
$$

We remark that (5.5) is the resolvent equation studied by Friedman and Shinbrot when $a(t, s)=-h^{\prime}(t-s) A(s)$ with $h(t)$ a scalar function and $\{A(t)\}$ a family of generators of analytic semigroups, cf. [4, Equation (1.5) and Theorem 3].

It is not clear what the appropriate definition of a solution of (IE) should be. Here we use one of the types of solutions considered by Grimmer and Miller [5, 6].

Definition 5.2. By a solution of (IE) is meant a function $x \in C([0, T], Y)$ for some $T>0$ such that (IE) is satisfied by $x(t)$ for $0 \leqslant t \leqslant T$.

While this seems the best definition for our purposes here, other definitions might be more appropriate in certain instances. In $[5,6]$, the concept of $L^{p}$-solution appeared to be more natural as was the concept of $L^{p}$ well-posedness.

THEOREM 5.3. Assume the hypotheses of the previous theorem are valid. Further, assume $f \in C\left([0, T], Y_{1}\right)$. Then

$$
x(t)=f(t)-\int_{0}^{t} r(t, u) f(u) d u
$$

is a solution of (IE).

Proof. Using (5.3) one obtains

$$
\begin{aligned}
\omega(t) & =\int_{0}^{t} r(t, u) f(u) d u \\
& =-\int_{0}^{t} a(t, u) f(u) d u+\int_{0}^{t} \int_{u}^{t} a(t, v) r(v, u) f(u) d v d u \\
& =-\int_{0}^{t} a(t, u) f(u) d u+\int_{0}^{t} \int_{0}^{v} a(t, v) r(v, u) f(u) d u d v
\end{aligned}
$$

and, as $a$ is closed,

$$
=-\int_{0}^{t} a(t, u) f(u) d u+\int_{0}^{t} a(t, v) \omega(v) d v .
$$


Thus, if $x(t)=f(t)-\omega(t), x(t)=\int_{0}^{t} a(t, u) x(u) d u+f(t)$. Also, as noted in the proof of the previous theorem $r(t, s)$ maps $Y_{1}$ into $Y$ and $r(t, s) x_{0}$ is strongly continuous in $Y$ for $x_{0} \in Y_{1}$. Hence, $\omega$ and $x$ are in $C([0, T], Y)$.

We remark that this result does not require $f$ to be differentiable which would be required if we attempted to use $R(t, s)$, the resolvent obtained from the differentiated version of (IE), as in Friedman and Shinbrot [4]. Also, the existence of solutions in Grimmer and Miller $[5,6]$ required differentiability of $f$. More differentiability is required of $a(t, s)$ in our context, however, than in $[5,6]$.

THEOREM 5.4. Assume the hypotheses of Theorem 5.1 are valid. Suppose $f \in$ $C([0, T], Y)$ and $x$ is a solution of (IE) with $x \in C\left([0, T], Y_{1}\right)$. Then on $[0, T], x(t)$ satisfies (5.6).

Proof. As $x \in C\left([0, T], Y_{1}\right), r(t, u) x(u)$ is continuous on $[0, t]$ into $Y$ and $X$. Also, $r(t, u) f(u)$ is continuous on $[0, t]$ into $X$. Now from (IE),

$$
\int_{0}^{t} r(t, u) x(u) d u-\int_{0}^{t} r(t, u) f(u) d u=\int_{0}^{t} r(t, u) \int_{0}^{u} a(u, s) x(s) d s d u .
$$

Let us now recall how $r(t, u)$ was obtained. Let $i: X \rightarrow X \times \mathcal{F}$ be the injection $x \rightarrow(x, 0)$ and $\Pi$ be the projection $\Pi(x, f)=x$. Then $r(t, s) x=(\partial / \partial s) R(t, s) x=$ $-\Pi U(t, s) C(s) i x$ for $x \in Y$. As $\Pi, U$, and $i$ are bounded operators, $C(s)$ is closed, and $x \in C\left([0, T], Y_{1}\right)$,

$$
\begin{aligned}
\int_{0}^{t} r(t, u) \int_{0}^{u} a(u, s) x(s) d s d u & =\int_{0}^{t} \int_{0}^{u} r(t, u) a(u, s) x(s) d s d u \\
& =\int_{0}^{t} \int_{s}^{t} r(t, u) a(u, s) x(s) d u d s
\end{aligned}
$$

and by (5.2)

$$
=\int_{0}^{t} r(t, s) x(s) d s+\int_{0}^{t} a(t, s) x(s) d s
$$

Thus,

$$
-\int_{0}^{t} r(t, u) f(u) d u=\int_{0}^{t} a(t, s) x(s) d s
$$

and (5.6) is seen to be valid.

As an illustration of the different resolvents involved with an integral equation such as (IE) consider the problem

$$
x(t, s)=\int_{0}^{t}(\partial / \partial s) x(u, s) d u+f(t, s) .
$$

Here we take $X=B U([0, \infty), R)$, the real valued bounded uniformly continuous functions with the sup norm and $a(t, v)=(\partial / \partial s)$ is constant. Clearly, the resolvent $R(t)$ is the translation semigroup and $r(t)$ is $-(\partial / \partial s) R(t)$. If $f(t, s)=f(t) g(s)$ with $g \in D(\partial / \partial s)$ and $f$ continuous, the use of $r$ and (5.6) yields

$$
x(t, s)=f(t) g(s)+\int_{0}^{t} f(u) g^{\prime}(s+t-u) d u
$$


which need not be differentiable, that is $x \notin Y$. In general, we see that we need $g$ to be twice differentiable so that the hypotheses of Theorem 5.3 are valid, that is $f(t, \cdot) \in C\left([0, T], Y_{1}\right)$.

If, however, $f$ is differentiable, the resolvent $R$ may be used to obtain

$$
x(t, s)=f(0) g(s+t)+\int_{0}^{t} f^{\prime}(u) g(s+t-u) d u
$$

which is a solution because $d(f(t) g(s)) / d t \in C([0, T], Y)$ using Theorem 2.7.

In general we see that (IE) has a solution if $f \in C\left([0, T], Y_{1}\right), f(0) \in Y$ and $f \in C^{1}([0, T], Y)$ or $f \in C^{2}([0, T], X)$, these cases corresponding to Theorem 5.3, Theorem 2.7, and Corollary 3.8, respectively.

\section{REFERENCES}

1. G. Chen, Control and stabilization for the wave equation in a bounded domain, SIAM J. Control 17 (1979), 66-81.

2. G. Chen and R. Grimmer, Semigroups and integral equations, J. Integral Equations 2 (1980), $133-154$.

3. __ Integral equations as evolution equations, J. Differential Equations (to appear).

4. A. Friedman and M. Shinbrot, Volterra integral equations in Banach space, Trans. Amer. Math. Soc. 126 (1967), 131-179.

5. R. C. Grimmer and R. K. Miller, Existence, uniqueness and continuity for integral equations in a Banach space, J. Math. Anal. Appl. 57 (1977), 429-447.

6. $201-216$. , Well posedness of Volterra integral equations in Hilbert space, J. Integral Equations 1 (1979),

7. R. C. Grimmer and G. Seifert, Stability properties of Volterra integrodifferential equations, J. Differential Equations 19 (1975), 142-166.

8. S. I. Grossman and R. K. Miller, Perturbation theory for Volterra integrodifferential systems, J. Differential Equations 8 (1970), 457-474.

9. M. E. Gurtin and A. C. Pipkin, A general theory of heat conduction with finite wave speeds, Arch. Rational Mech. Anal. 31 (1968), 113-126.

10. K. B. Hannsgen, The resolvent kernel of an integrodifferential equation in Hilbert space, SIAM J. Math. Anal. 7 (1976), 481-490.

11. __ Uniform $L^{1}$ behavior for an integrodifferential equation with parameter, SIAM J. Math. Anal. 8 (1977), 626-639.

12. T. Kato, Linear evolution equations of "hyperbolic" type, J. Fac. Sci. Univ. Tokyo Sec. I 17 (1970), $241-258$.

13. ___ Linear evolution equations of "hyperbolic" type. II, J. Math. Soc. Japan 25 (1973), 648-666

14. R. K. Miller, Volterra integral equations in a Banach space, Funkcial. Ekvac. 18 (1975), 163-193.

15. An integrodifferential equation for rigid heat conductors with memory, J. Math. Anal. Appl. 66 (1978), 313-332.

16. ___ Nonlinear Volterra integral equations, Benjamin, Menlo Park, Calif., 1971.

17. R. K. Miller and R. L. Wheeler, Asymptotic behavior for a linear Volterra integral equation in Hilhert space, J. Differential Equations 23 (1977), 270-284.

18. W_ Well-posedness and stabiltiy of linear Volterra integrodifferential equations in abstract spaces, Funkcial. Ekvac. 21 (1978), 279-305.

19. A. Pazy, Semigroups of linear operators and applications to partial differential equations, Dept. Math. Lecture Note \# 10, University of Maryland, 1974.

20. A. J. Pritchard and J. Zabczyk, Stability and stabilizability of infinte dimensional systems, SIAM Rev. 23 (1981), 25-52.

21. H. Tanabe, Equations of evolution, Pittman, London, 1979.

Department of Mathematics, Southern Illinois University, Carbondale, Illinois 62901 